\title{
Design and Control of a Parallel Robot for Mold Polishing
}

\author{
Takashi Harada ${ }^{1, a}$ \\ ${ }^{1}$ Kinki University, Faculty of Science and Engineering, 3-4-1 Kowakae Higashiosaka, 5778502, Osaka, Japan
}

\begin{abstract}
A novel redundantly actuated parallel robot for mold polishing work is proposed. Cogging-less direct drive motors are implemented in the prototype. Instead of using conventional spherical sliding bearings, ball bearings with appropriate preload are implemented in the rolling pairs of the prototype. These mechanical parts enable fine force control for mold polishing work. The rated force of the robot perpendicular to the surface on the workpiece is $20 \mathrm{~N}$ with a tangential force of $5 \mathrm{~N}$, which enable the robot to execute polishing tasks as well as skilled workers. In addition, the polishing velocity along the tangential direction is $60 \mathrm{~m} / \mathrm{min}$, which exceeds skilled workers' velocity of $10 \mathrm{~m} / \mathrm{min}$. High speed feed contributes to reducing the polishing force and improving the quality of the surface of the mold. Kinematics, statics, dynamics and control of redundantly actuated parallel robot are discussed in this paper. Impedance control was implemented to the prototype for stably executing the mold polishing work.
\end{abstract}

\section{Introduction}

In the mold polishing work, operator oscillates a stick type grinding stone on the surface of the work piece by hand at approximately speed of $10 \mathrm{~m} / \mathrm{min}$, with from 20 $\mathrm{N}$ to $30 \mathrm{~N}$, up to $50 \mathrm{~N}$ contacting force. More than several hundreds of reciprocates oscillating is needed for fine finishing. There have been a lot of requests for automating this heavy work, and several researches for automating the mold polishing work using industrial robot manipulators[1-5]. Industrial robots are aimed not for force control but for position control. Frictions in the gear trains for enlarge the motor torque looses the back drivability and disturbs the fine force generation. Force feedback or mechanical floating devices has been tested for mold polishing by conventional industrial robot. However, industrial robot, consisted by serial mechanisms include heavy motors, has large effective mass at the tip, that disturbs high speed oscillating motion for mold polishing. Conventional researches polishing by industrial robot use rotating polishing tools for increasing the polishing speed. The characteristics of the finished surface by the rotating polishing tool is differs from that by the human operator using stick grinding stone by hand. Recently, high speed industrial robots, consisted by parallel mechanisms, have been commercially available [6,7]. Parallel robots have sufficient speed for the mold polishing tasks. However, these robots are not suitable when the robots contact their environment because almost all parallel robots are aimed not for force control but for position control.

In this paper, a novel redundantly actuated DALTA type [8] parallel robot aimed for force control is proposed. Kinematics, statics, dynamics, control and prototyping of

\footnotetext{
a Corresponding author: harada@mech.kindai.ac.jp
}

the parallel robot are discussed. Impedance control was implemented to the prototype for stably executing the mold polishing work.

\section{Design of the parallel robot}

\subsection{Actuators}

For the cogging-less fine force control, BLC09-25 by ShinMaywa Industries, Ltd., built in type slot-less direct drive motor with rated torque of $0.6 \mathrm{Nm}$, is selected. High resolution rotary encoder, Tonic by RENISHAW plc with $1,184,000$ plus/rev was installed to the actuator. Instead of friction-full cross roller bearing, small friction deep groove ball bearings are installed around the motor. Appropriate pre pressure is applied to the bearings, for removing the clearances in the bearings. Developed actuator is shown in Fig. 1. Specifications of the actuator are summarized in Table 1.

\subsection{Parallelogram linkages}

Small friction parallelogram linkages with rotating pairs by ball bearings are designed for the robot. Small springs are installed in the rotating pairs for removing clearance, at the same time, for releasing unexpected force around the over constrained parallelogram linkage.

\subsection{Design of the length of the arm}

In order to gain the small torque of the slot-less motor, redundantly actuation of 4 motors for 3 dof parallel robot are adopted. Long arm enables high speed and large 
workspace of the robot, but at the same time, transmits small force to the moving plate. Length of the arm is designed in $50 \mathrm{~mm}$ for appropriate workspace and sufficient generative fore and velocity at the moving plate (MP). Specification of the robot are summarized in Table 2. Schematic image of the mold polishing work by the robot and picture of the prototype are shown in Fig. 2.

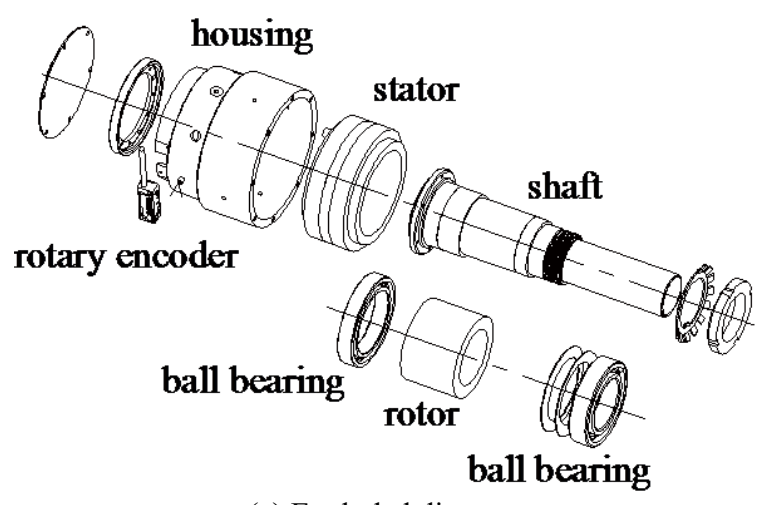

(a) Exploded diagram

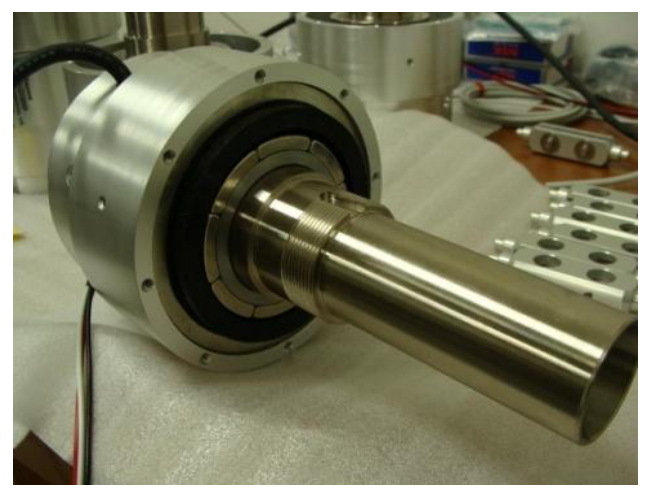

(b) Prototype

Figure 1. Direct drive motor.

Table 1. Specifications of the actuator.

\begin{tabular}{|c|r|}
\hline Rated torque & $0.527[\mathrm{Nm}]$ \\
\hline Peak torque & $0.924[\mathrm{Nm}]$ \\
\hline Rated speed & $100\left[\mathrm{~min}^{1}\right]$ \\
\hline Resolition & $1,184 \mathrm{k}[\mathrm{p} / \mathrm{rev}]$ \\
\hline
\end{tabular}

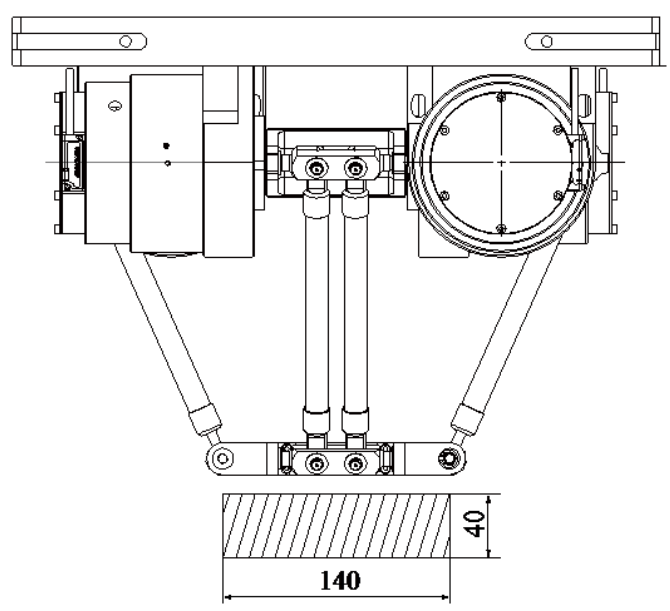

(a) Effective workspace

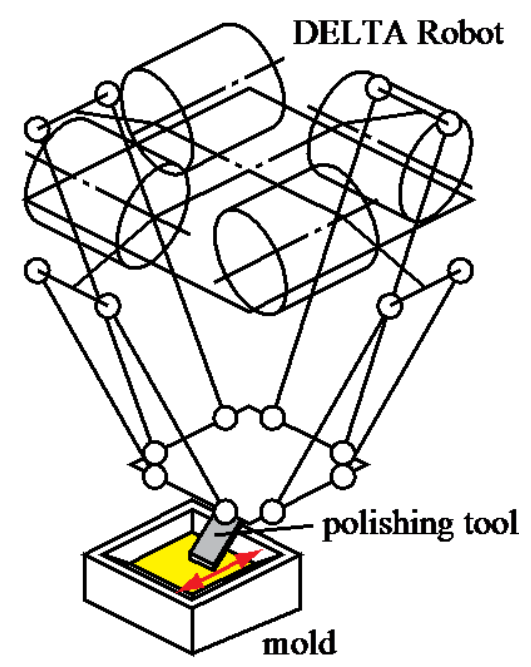

(b) Schematic image of mold polishing

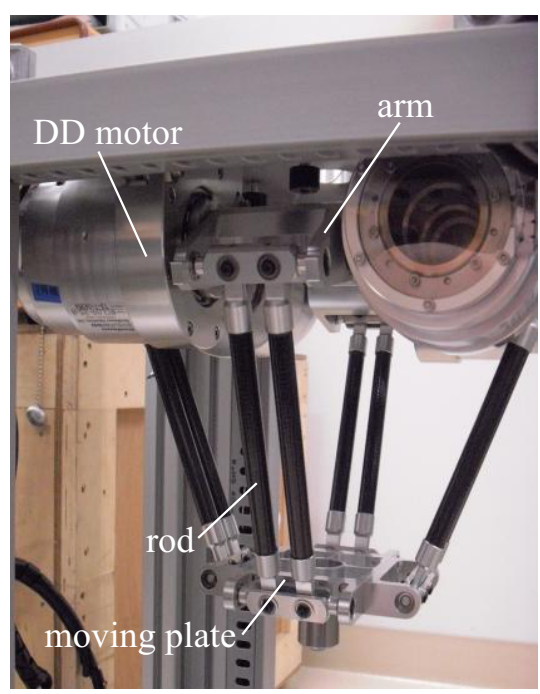

(b) Prototype

Figure 2. Mold polishing robot. 
Table 2. Specifications of the robot.

\begin{tabular}{|c|c|}
\hline length of arm & $50[\mathrm{~mm}]$ \\
\hline length of rod & $200[\mathrm{~mm}]$ \\
\hline rated polishing force & $20[\mathrm{~N}]$ \\
\hline rated speed $(X Y)$ & $60[\mathrm{~m} / \mathrm{min}]$ \\
\hline work space (XY) & $140 \times 140[\mathrm{~mm}]$ \\
\hline$(\mathrm{Z})$ & $40[\mathrm{~mm}]$ \\
\hline
\end{tabular}

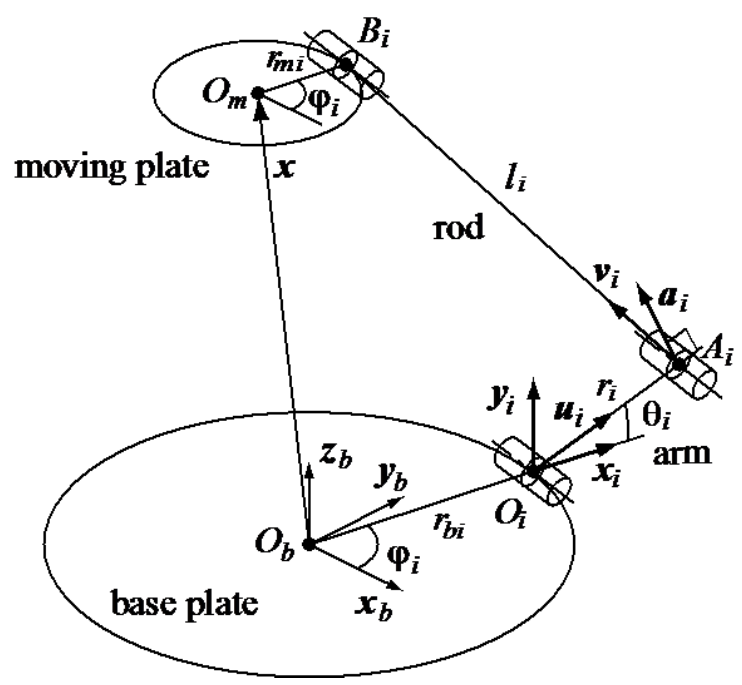

$O_{b}:$ Origin of the base plate(BP)

$O_{m}:$ Origin of the moving plate(MP)

$O_{i}$ : Position of the ith pair between the ith link and the BP

$A_{i}$ : Position of the ith pair between the ith link and the rod

$B_{i}$ : Position of the ith pairbetween the ith link and the MP

$r_{b i}$ : Radius of the BP

$r_{m i}:$ Radius of the MP

$\varphi_{i}:$ Angle of location of the ith pair on the MP

$x$ : Position of the MP w.r.t. The BP coordinates

$\boldsymbol{u}_{i}$ : Unit direction vector of the arm

$r_{i} \quad$ : length of the arm

$v_{i}:$ Unit direction vector of the rod

$l_{i} \quad$ : length of the rod

$\theta_{i}:$ Angle of the motor

$\boldsymbol{x}_{i}, \boldsymbol{y}_{i}$ : Unit direction vectors perpendicular to the axis of the motor

Figure 3. Kinematic model of the robot.

\section{Robot Control for Mold Polishing}

\subsection{Position Analysis}

Kinematic model of the parallel robot is shown in Fig.3. There are two routes from $O_{i}$ to $B_{i}$ as shown in Eq. (1) and Eq. (2).

$$
\begin{aligned}
\overrightarrow{O_{i} B_{i}}= & \overrightarrow{O_{i} O_{b}}+\overrightarrow{O_{b} O_{m}}+\overrightarrow{O_{m} B_{i}}=-r_{b i} \boldsymbol{s}_{i}+\boldsymbol{x}+r_{m i} \boldsymbol{s}_{i} \\
\boldsymbol{x}= & {\left[\begin{array}{lll}
x & y & z
\end{array}\right]^{T} } \\
\boldsymbol{s}_{i}= & {\left[\begin{array}{lll}
\cos \varphi_{i} & \sin \varphi_{i} & 0
\end{array}\right]^{T}, i=1, \ldots, 4 } \\
& \overrightarrow{O_{i} B_{i}}=\overrightarrow{O_{i} A_{i}}+\overrightarrow{A_{i} B_{i}}=r_{i} \boldsymbol{u}_{i}+l_{i} \boldsymbol{v}_{i} \\
\boldsymbol{u}_{i}= & \cos \theta_{i} \boldsymbol{x}_{i}+\sin \theta_{i} \boldsymbol{y}_{i} \\
\boldsymbol{x}_{i}= & {\left[\begin{array}{lll}
\cos \varphi_{i} & \sin \varphi_{i} & 0
\end{array}\right]^{T} } \\
\boldsymbol{y}_{i}= & {\left[\begin{array}{lll}
0 & 0 & 1
\end{array}\right]^{T} }
\end{aligned}
$$

By equation Eq. (1) and (2), one obtains loop closure equation that relates angle of the motor $\theta_{i}$ and position of the MP $\boldsymbol{x}$.

$$
\boldsymbol{x}-\left(r_{b i}-r_{m i}\right) \boldsymbol{s}_{i}=r_{i} \boldsymbol{u}_{i}+l_{i} \boldsymbol{v}_{i}
$$

\subsection{Jacobian Matrices}

Jacobian matrices $\boldsymbol{J}_{x}$ and $\boldsymbol{J}_{q}$ are given by differentiating both sides of Eq. (3) with respect to time as follows,

$$
\begin{aligned}
& \boldsymbol{J}_{x} \dot{\boldsymbol{x}}=\boldsymbol{J}_{q} \dot{\boldsymbol{q}} \\
& {\left[\begin{array}{c}
\boldsymbol{v}_{1}{ }^{T} \\
\vdots \\
\boldsymbol{v}_{4}{ }^{T}
\end{array}\right] \cdot\left[\begin{array}{c}
\dot{x} \\
\dot{y} \\
\dot{z}
\end{array}\right]=\left[\begin{array}{ccc}
\boldsymbol{v}_{1}{ }^{T} \boldsymbol{a}_{1} & & \boldsymbol{0} \\
& \ddots & \\
\boldsymbol{0} & & \boldsymbol{v}_{4}{ }^{T} \boldsymbol{a}_{4}
\end{array}\right]\left[\begin{array}{c}
r_{1} \dot{\theta}_{1} \\
\vdots \\
r_{4} \dot{\theta}_{4}
\end{array}\right]} \\
& \boldsymbol{a}_{i}=\frac{d \boldsymbol{u}_{i}}{d \theta_{i}}=-\sin \theta_{i} \boldsymbol{x}_{i}+\cos \theta_{i} \boldsymbol{y}_{i}
\end{aligned}
$$

\subsection{Statics}

Relationship between the force $f_{x}$ at the MP and the torques of the motors $f_{q}$ is given as,

$$
\begin{aligned}
& \boldsymbol{f}_{x}=\boldsymbol{J}^{T} \boldsymbol{f}_{q} \\
& \boldsymbol{J}=\boldsymbol{J}_{q}^{-1} \boldsymbol{J}_{x}, \\
& \boldsymbol{f}_{x}=\left[\begin{array}{lll}
f_{x} & f_{y} & f_{z}
\end{array}\right]^{T} \\
& \boldsymbol{f}_{q}=\left[\begin{array}{lll}
f_{q 1} & \cdots & f_{q 4}
\end{array}\right]^{T}=\left[\begin{array}{lll}
\frac{\tau_{1}}{r_{1}} & \cdots & \frac{\tau_{4}}{r_{4}}
\end{array}\right]^{T}
\end{aligned}
$$

In Eq. (5), torque of each motor $\tau_{i}$ is converted to the generative force of the actuator $f_{q i}$ which corresponds to the force at $A_{i}$ to the direction of $\boldsymbol{a}_{i}$ in Eq. (4). In case of DELTA robot, the Jacobian matrix $\boldsymbol{J}_{q}$ becomes diagonal. Its inverse is also diagonal matrix, each element of $\boldsymbol{J}_{q}^{-1}$ is equal to the reciprocal of corresponding element of $\boldsymbol{J}_{q}$. Jacobian matrix $\boldsymbol{J}$ is given as the following simple form.

$$
\begin{aligned}
\boldsymbol{J} & =\boldsymbol{J}_{q}{ }^{-1} \boldsymbol{J}_{x}=\left[\begin{array}{lll}
\frac{\boldsymbol{v}_{1}}{\boldsymbol{v}_{1}^{T} \boldsymbol{a}_{1}} & \cdots & \frac{\boldsymbol{v}_{4}}{\boldsymbol{v}_{4}{ }^{T} \boldsymbol{a}_{4}}
\end{array}\right] \\
& =\left[\begin{array}{lll}
\boldsymbol{c}_{1} & \cdots & \boldsymbol{c}_{4}
\end{array}\right]
\end{aligned}
$$

From the $1^{\text {st }}$ equation of Eq. (5), actuator fore $\boldsymbol{f}_{q}$ is given as, 


$$
\boldsymbol{f}_{q}=\left(\boldsymbol{J}^{T}\right)^{+} \boldsymbol{f}_{x}+\boldsymbol{s} f_{c}
$$

$\left(\boldsymbol{J}_{T}\right)^{+}$represents pseudo-inverse of $\boldsymbol{J}^{T}, \boldsymbol{s}$ represents $4 \times 1$ projection vector which gives the orthogonal complement of $\boldsymbol{J}^{T}, f_{c}$ is an arbitrary constant which gives amount of the internal force of redundantly actuation.

\subsection{Impedance control}

Mold polishing work by the robot is done by the impedance control $[9,10]$. Here, we derive the force-based impedance control of the redundantly actuated parallel robot. The equation of motion of the parallel robot can be rewritten as follows:

$$
f=M(x) \ddot{x}+h(x, \dot{x})+g(x)
$$

The objective of impedance control is to control the dynamical behavior of the MP according to

$$
\begin{aligned}
& \boldsymbol{f}_{d}=\boldsymbol{M}_{d} \ddot{\boldsymbol{x}}_{e}+\boldsymbol{B}_{d} \dot{\boldsymbol{x}}_{e}+\boldsymbol{K}_{d} \boldsymbol{x}_{e} \\
& \boldsymbol{x}_{e}=\boldsymbol{x}_{f}-\boldsymbol{x}
\end{aligned}
$$

Vectors $\boldsymbol{x}_{f}$ and $\boldsymbol{x}$ are the reference trajectory and actual trajectory of the MP, respectively. $\boldsymbol{M}_{d}, \boldsymbol{B}_{d}$, and $\boldsymbol{K}_{d}$ are constant matrices that define the impedance characteristics of mass, damping, and stiffness, respectively. The actual position $\boldsymbol{x}$ are calculated via the forward kinematics from the positions of actuators. The force command $\boldsymbol{f}_{e}$ that can yield the desired impedance at the MP is expressed as follows:

$$
\boldsymbol{f}_{e}=\boldsymbol{f}_{d}+\widehat{\boldsymbol{M}}(\boldsymbol{x}) \ddot{\boldsymbol{x}}+\widehat{\boldsymbol{h}}(\boldsymbol{x}, \dot{\boldsymbol{x}})+\widehat{\boldsymbol{g}}(\boldsymbol{x})
$$

the where $\hat{\boldsymbol{M}}, \hat{\boldsymbol{h}}$, and $\hat{\boldsymbol{g}}$ are the nominal values of $\boldsymbol{M}, \boldsymbol{h}$, and $g$, respectively. The force command for the actuators is given by (7) as follows:

$$
\boldsymbol{f}_{q}=\left(\boldsymbol{J}^{T}\right)^{+} \boldsymbol{f}_{e}+\boldsymbol{s} f_{c}
$$

\section{Experiments}

\subsection{Experimental Setup}

Polishing stone is attached at the MP via 3 axes force sensor. Hexapod, 6 dof Stewart platform parallel robot, and rotary table are prepared for tilting, panning and rotating the work piece. iBIS by MTT Corp., DSP based real time controller equipped with $\mathrm{D} / \mathrm{A}, \mathrm{A} / \mathrm{D}, \mathrm{PiO}$ and counter, is used for control the mold polishing parallel robot. A model based control software was developed by Simulink. iBIS can communicate to the host computer during executing the polishing work, positions, velocities, accelerations and forces are monitored and stored to the host computer. In addition, polishing parameter, such as desired trajectory, velocity, impedance, polishing force, etc, can be changed during executing the polishing work. Control system of the mold polishing robot is shown in Fig. 4. Experimental setup of the mold polishing is shown in Fig. 5.

\subsection{Mold Polishing by Robot System}

Mold polishing work was executed by the parallel robot. For the first test, flat surface was polished with polishing speed of $10 \mathrm{~m} / \mathrm{min}$ (same velocity as human operator), and $20 \mathrm{~m} / \mathrm{min}$ as shown in Fig. 6 (a). Actual polishing force and velocity were monitored by the host computer as shown in Fig. 6 (b). Movie of the test is uploaded to our website [11].

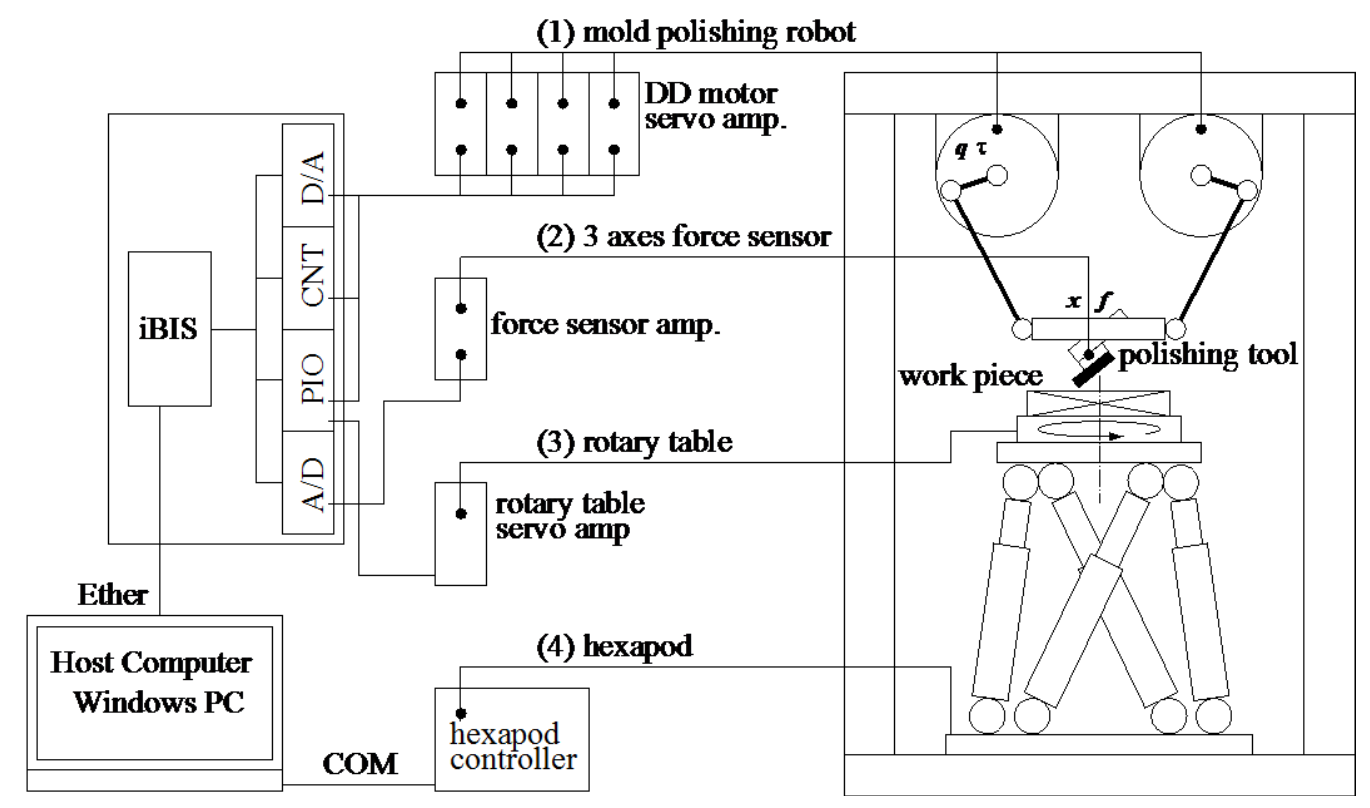

Figure 4. Control system of the mold polishing robot. 


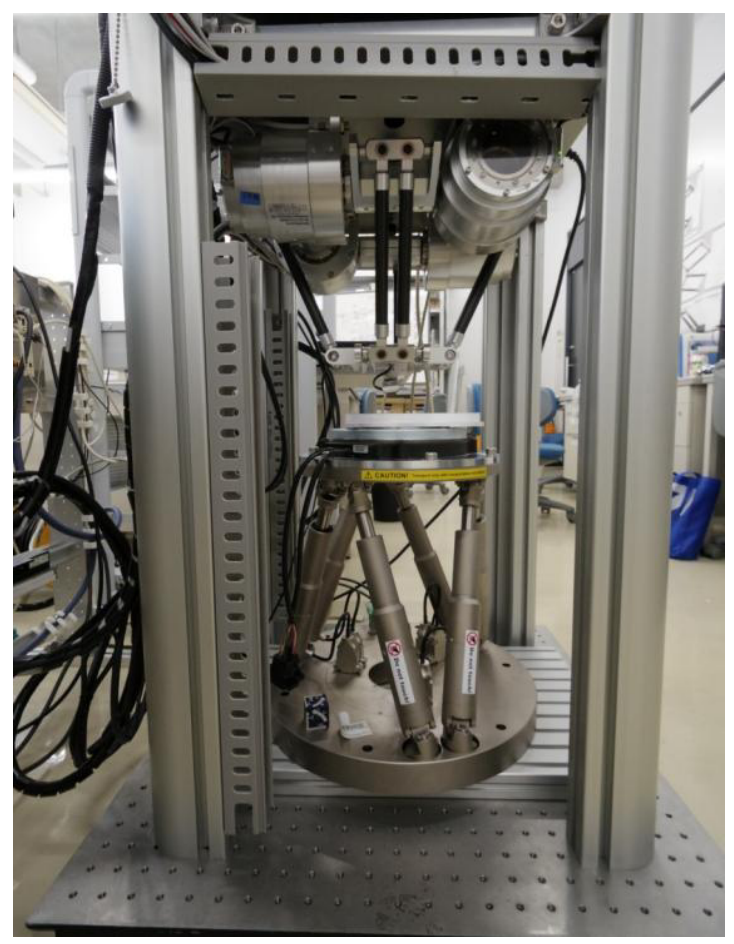

Figure 5. Experimental setup of the mold polishing.

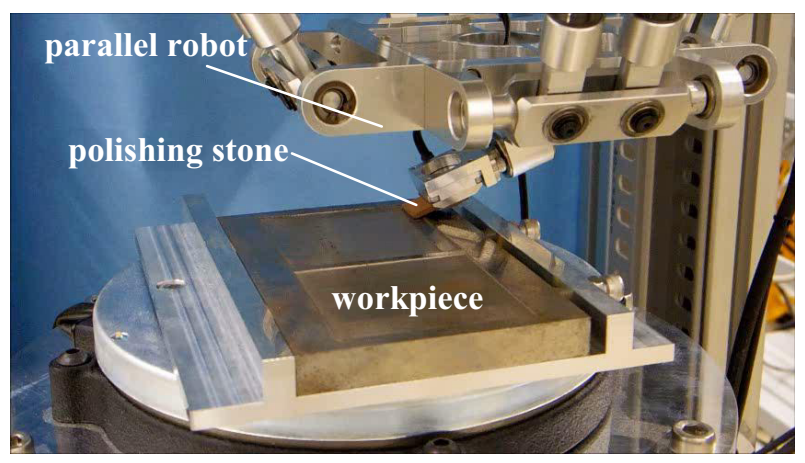

(a) polishing tool and workpiece

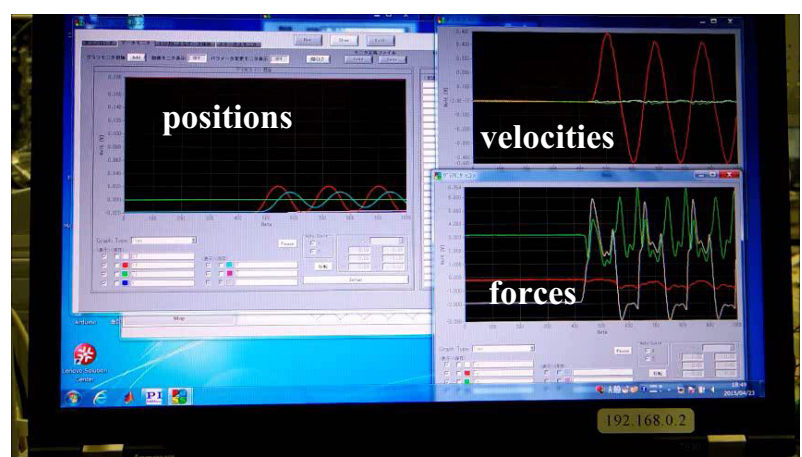

(b) monitoring the polishing work

Figure 6. Experimental setup of the mold polishing.

\section{Summary}

A novel redundantly actuated parallel robot for mold polishing work was proposed. Kinematics, statics, dynamics and control of redundantly actuated parallel robot were discussed in this paper. Impedance control was implemented to the prototype for stably executing the mold polishing work. Optimizing the polishing conditions and polishing of the curved surface are our future researches.

\section{Acknowledgement}

This work was supported by MEXT-supported program for the strategic Research Foundation to Private Universities 2012-2014., and JSPS KAKENHI Grant-inAid for Scientific Research (C) Grant Number 15K05918.

\section{References}

1. L. Pilny and G. Bissacco, Development of on the machine process monitoring and control strategy in Robot Assisted Polishing, CIRP Annals - Manu. Tech., 64, 313-316 (2015)

2. K. Raju and B. Reddy, Design and Simulation of Die Casting Mould using Robot Path, International Journal of Innovative Research and Development, 4(8), 29-34 (2015)

3. K. Shibuya and S. Issiki, Evaluation of Metallic Mold Surfaces Polished by an Industrial Robot with Stick Whetstones, Int. J. of Automation Technology, 8(2), 253-263 (2014)

4. F. Nagata, et. al., CAD/CAM-based position/force controller for a mold polishing robot, Mechatronics, 17(4-5), 207-216 (2007)

5. M.C. Lee1, et. al., A robust trajectory tracking control of a polishing robot system based on CAM data, Rob. and Computer-Integrated Manufacturing, 17(1-2), 177-183 (2001)

6. W. Tsai L, Robot Analysis , Wiley Interscience, (1999)

7. J. P. Merlet, Parallel Robots, Springer, (2006)

8. R. Clavel, R, DELTA, a fast robot with parallel geometry, Proc. Int. Symposium on Industrial Robots, 91-100 (1988)

9. N. Hogan, "Impedance Control: An Approach to Manipulation: Part I-III," Transactions of the ASME, Journal of Dynamic Systems, Measurement, and Control, 107(1), 1-24 (1985)

10. T. Harada and M. Nagase, Impedance Control of a Redundantly Actuated 3-DOF Planar Parallel Link Mechanism Using Direct Drive Linear Motors, Proc. of IEEE ROBIO, 501-506 (2010)

11. https://www.youtube.com/watch?feature=player det ailpage $\& v=3 \mathrm{AcZ} 6 \mathrm{QRy} 3 \mathrm{RI}$ 